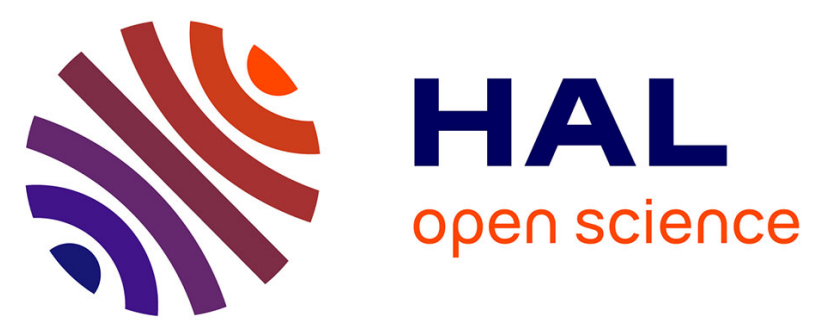

\title{
How seaweeds release the excess energy from sunlight to surrounding sea water
}

Kenichi Koizumi, Makoto Hatakeyama, Mauro Boero, Katsuyuki Nobusada, Hirokazu Hori, Taku Misonou, Shinichiro Nakamura

\section{- To cite this version:}

Kenichi Koizumi, Makoto Hatakeyama, Mauro Boero, Katsuyuki Nobusada, Hirokazu Hori, et al.. How seaweeds release the excess energy from sunlight to surrounding sea water. Physical Chemistry Chemical Physics, 2017, 19 (24), pp.15745-15753. 10.1039/C7CP02699D . hal-02991570

\author{
HAL Id: hal-02991570 \\ https://hal.science/hal-02991570
}

Submitted on 4 Dec 2020

HAL is a multi-disciplinary open access archive for the deposit and dissemination of scientific research documents, whether they are published or not. The documents may come from teaching and research institutions in France or abroad, or from public or private research centers.
L'archive ouverte pluridisciplinaire HAL, est destinée au dépôt et à la diffusion de documents scientifiques de niveau recherche, publiés ou non, émanant des établissements d'enseignement et de recherche français ou étrangers, des laboratoires publics ou privés. 


\title{
How seaweeds release the excess energy from
}

\section{sunlight to surrounding sea water}

\author{
Kenichi Koizumi, ${ }^{\dagger}$ Makoto Hatakeyama, ${ }^{\mathbb{I I}}$ Mauro Boero, ${ }^{\S}$ Katsuyuki Nobusada, ${ }^{*, \dagger}$ \\ Hirokazu Hori, "Taku Misonou, ${ }^{\text {and Shinichiro Nakamurall }}$ \\ Department of Theoretical and Computational Molecular Science, Institute for Molecular \\ Science, Myodaiji, Okazaki 444-8585, Japan, Elements Strategy Initiative for Catalysts and \\ Batteries (ESICB), Kyoto University, Katsura, Kyoto 615-8520, Japan, Nakamura Laboratory, \\ RIKEN Innovation Center, 2-1 Hirosawa, Wako, Saitama 351-0198, Japan, Institut de Physique et \\ Chimie des Matériaux de Strasbourg UMR 7504, University of Strasbourg and CNRS, 23 rue du \\ Loess, F-67034 Strasbourg, France, Faculty of Life and Environmental Sciences, Graduate \\ School of University of Yamanashi 4-4-37, Takeda, Kofu, Yamanashi, 400-8510 Japan, and \\ Computational Chemistry Applications Unit, Advanced Center for Computing and \\ Communication, RIKEN,2-1, Hirosawa, Wako, Saitama 351-0198, Japan \\ E-mail: nobusada@ims.ac.jp
}

\footnotetext{
Abstract

${ }^{*}$ To whom correspondence should be addressed

${ }^{\dagger}$ Department of Theoretical and Computational Molecular Science, Institute for Molecular Science, Myodaiji, Okazaki 444-8585, Japan

ॠElements Strategy Initiative for Catalysts and Batteries (ESICB), Kyoto University, Katsura, Kyoto 615-8520, Japan

IINakamura Laboratory, RIKEN Innovation Center, 2-1 Hirosawa, Wako, Saitama 351-0198, Japan

${ }^{\S}$ Institut de Physique et Chimie des Matériaux de Strasbourg UMR 7504, University of Strasbourg and CNRS, 23 rue du Loess, F-67034 Strasbourg, France

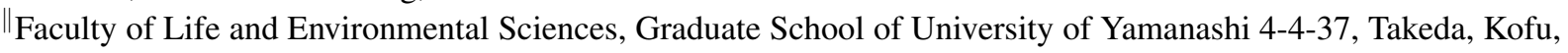
Yamanashi, 400-8510 Japan

${ }^{\perp}$ Computational Chemistry Applications Unit, Advanced Center for Computing and Communication, RIKEN,2-1, Hirosawa, Wako, Saitama 351-0198, Japan
} 
We report an atomistic insight into the mechanism regulating the energy released by a porphyra-334 molecule, the ubiquitous photosensitive component of marine algae, in a liquid water environment upon an electron excitation. To quantify this rapidly occurring process, we resort to the Fourier analysis of the mass-weighted auto-correlation function, providing evidence for a remarkable dynamical change in the number of hydrogen bonds among water molecules and between the porphyra-334 and its surrounding hydrating water. Hydrogen bonds between the porphyra-334 and close by water molecules can act directly and rather easily to promote an efficient transfer of the excess kinetic energies of the porphyra-334 to the surrounding solvating water molecules via an activation of the collective modes identified as hydrogen-bond stretching modes in liquid water which eventually results in a disruption of the hydrogen bond network. Since porphyra-334 is present in seaweeds, aquatic cyanobacteria (blue-green algae) and red algae, our findings allow to address the question how algae in oceans or lakes, upon sunlight absorption, can release large amounts of energy into surrounding water without destabilizing neither their own nor the $\mathrm{H}_{2} \mathrm{O}$ molecular structure.

\section{Introduction}

Sunlight is the primary energy source of living organisms on the earth. and, in this context, photosynthesis is one of the most important metabolism processes. It consists in the synthesis of nutrients from the environmental carbon dioxide and water, catalyzed by visible sunlight. Yet, the solar spectrum is wider than the simple visible light and includes also high-energy ultra-violet (UV) radiation (UVR), harmful to any living organism. Indeed, UVR can damage to various extents biomolecules and, specifically, nucleic acids (DNA and RNA) in the cell through direct and indirect pathways. To cope with this problem, several evolution strategies have been adopted by nature to protect life from the UVR damage. A clear understanding of these strategies is now becoming increasingly important also for the sunlight-exposed biosphere because of the constant increasing of UVR on Earth, particularly enhanced in the last decades, resulting from the disruption of the ozone layer in the stratosphere mainly due to an anthropogenic factor. 
The development and accumulation of UV absorbing substances inside the cells, such as mycosporine-like amino acids (MAA), can be regarded as a powerful physiological solution and a fundamental step against UVR adopted by nature in general and algae in particular. ${ }^{1-3}$ MAA was found in a wealth of organisms, particularly $n$ green-blue ${ }^{4,5}$ and red algae, ${ }^{6}$ living in environment highly irradiated by sunlight and display a maximum absorption in the wavelength range between 300 and $350 \mathrm{~nm}$, thus absorbing mainly UV-A and UV-B. ${ }^{7}$ In fact, MAA is ubiquitous in various aquatic and terrestrial vegetal organisms, namely cyanobacteria, algae and fungi; moreover it is also present in some aquatic animals. ${ }^{8}$ The function exerted by MMA extends beyond that of an UV protector, and includes also other physiological roles as an antioxidant, an osmolyte and as an agent preventing desiccation and thermal stress. ${ }^{9-11}$ This rich variety of functions make MMA a fundamental compound for life in general and its study a fundamental multidisciplinary target from photocatalysis to advanced biomaterials. Concerning its primary function, the UV absorption characteristics of MAA have been extensively studied with respect to their physiological and ecological importance. Instead, research activities focused more on the physico-chemical aspects and energy transfer mechanisms of MAA molecules are rare and still at a pioneering stage. Yet, the efficiency of MMA in energy adsorption upon UVR is remarkable and worthy of the attention of research efforts at the crossroad among physics, chemistry and biology. In fact, the molar extinction coefficient of MMA can reach extraordinary values up to a range $28000-50000 \mathrm{M}^{-1} \mathrm{~cm}^{-1}$ with unsurpassed sunscreen properties. ${ }^{12,13}$ Among the studies available to date, Misonou and coworkers ${ }^{14}$ have shown that MAA is able to quench the excited state of a pyrimidine nucleotide, a typical component of nucleic acids. While MAA is known to possess a high stability even under relatively strong UV irradiation, ${ }^{15}$ the absorbed energy is expected to be dissipated very rapidly in the surrounding water environment, ${ }^{14,16-18}$ although the mechanism responsible for this dissipation is largely unknown. Furthermore, MAA has a low level of fluorescence $\left(1 \times 10^{-4}\right),{ }^{16-19}$ a fact that makes hard unraveling the mechanism via experimental probes.

The scope of the present study is then to address the fundamental question of the excess energy dissipation and to shed some light into the basic mechanism responsible for this process. To this 
aim, we selected one specific and well assessed structure of MMA, among the over thirty existing species: The porphyra-334 system. Our investigations make use of first principles molecular dynamics (FPMD) simulations, ${ }^{20}$ nowadays regarded as a powerful tools to perform atomic-level virtual experiments suitable to unravel the dynamical processes occurring in condensed phases from aqueous solutions, ${ }^{21-25}$ to solid state chemistry, ${ }^{26-31}$ and to biosystems. ${ }^{32-35}$ For the specific problem on which this work is focused, the inclusion of the electronic structure and the possibility of following its dynamical evolution is essential to properly account for the possibility of chemical bond breaking and formation which could be involved in the energy release process, along with changes in the hydrogen bond (H-bond) structured network, induced strong polarizations and redistributions of atomic charges occurring during this type of processes. The quantum description of the system is based on the density functional theory ${ }^{36,37}$ (DFT) framework, allowing for a good compromise between accuracy and computational costs. Such an approach, backed by auxiliary quantum chemistry ab initio calculations, allow to clarify the fundamental scenario according to which an excited porphyra-334 molecule solvated in water can release an excess energy acquired upon electronic excitation and operate an efficient transfer to the $\mathrm{H}_{2} \mathrm{O}$ molecules of its own environment. This, in turn, provides a rather clear answer to the question about the non-destructive release of energy of seaweeds in sea water occurring upon sunlight adsorption.

\section{Computational Methods}

\section{Details of the Simulations}

Starting from a standard porphyra-334 molecular structure, we constructed a comprehensive set of configurations, reported in Figure 1, differing in the protonation state of the hydroxy groups, oxygen and nitrogen atoms. All these configurations were then optimized within a DFT approach in which the exchange-correlation interaction was described by a hybrid B3LYP ${ }^{38-40}$ functional. All these optimizations were done on the electronic ground state of the porphyra-334 system, in an attempt at unraveling their relative stability. This extensive search indicated that two of these ge- 
ometries are the most stable. Energetically they are rather close to each other and correspond to the configurations labeled as 2 and 8 in Figure 1. Details will be discussed in the next paragraph. After the determination of these two ground state stable configurations, we computed the corresponding excited-state geometries via a single-excitation configuration interaction (CIS) approach. In all these quantum chemical calculations, a localized 6-31G(p,d) basis sets ${ }^{41}$ was adopted and the effect of the solvating water was kept into account by the inclusion of a polarizable continuum model (PCM). ${ }^{42,43}$ For this set of static calculations, the GAUSSIAN09 package ${ }^{44}$ was used.

(a) Configuration 1

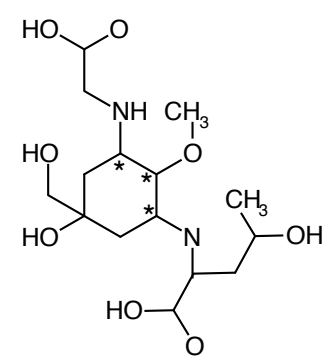

$-1258.1339$

(e) Configuration 5

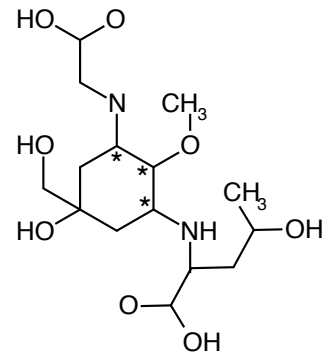

$-1258.1311$ (b) Configuration 2

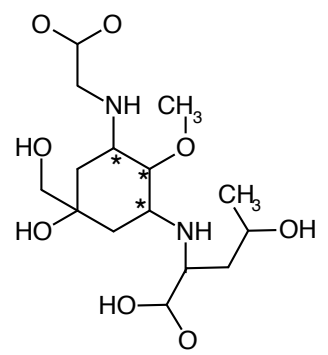

$-1258.1449$

(f)

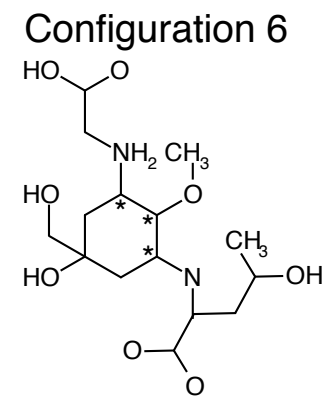

$-1258.0801$ (c) Configuration 3

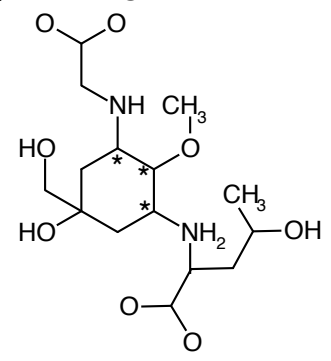

Unstable

(g) Configuration 7

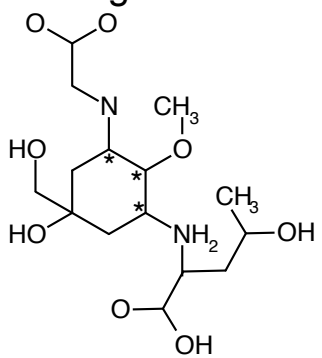

$-1258.1058$ (d) Configuration 4<smiles>COC1=C(NC(CC(C)O)C(=O)O)CC(O)(CO)CC1NCC(O)O</smiles>

Unstable

(h) Configuration 8<smiles>COC1=C(NC(CC(C)O)C(=O)O)CC(O)(CO)CC1NCC(O)O</smiles>

$-1258.1505$

Figure 1: The eight configurations of the porphyra-334 molecule used for ground state geometry optimizations and related total energies (a.u.). The label "Unstable" means that proton transfer processes were observed during the geometry optimization procedure. The configuration labeled as 3 can switch to configuration 2, as well as configuration 4 can change to configuration 8 upon optimization. The carbon atoms marked with asterisks inside the cyclohexane rings are linked to three atoms and carry either a double $(1.33 \AA$ ) or a single $(1.54 \AA$ ) bond.

All subsequent dynamical simulations were conducted at the FPMD level within the CarParrinello molecular dynamics (CPMD) ${ }^{45}$ method. Valence electrons wavefunctions were expanded on a plane-wave basis set with an energy cut-off of $80 \mathrm{Ry}$ and the sampling of the Brillouin 
zone was restricted to the $\Gamma$ point. All core-valence interactions were described by analytical norm-conserving pseudopotentials (PPs) of the Hartwigsen-Goedecker-Hutter (HGH) type. ${ }^{47-49}$ The generalized gradient approximation (GGA) adopted in all FPMD simulations is the one composed of the Becke exchange ${ }^{39}$ and the Lee-Yang-Parr-correlation ${ }^{40}$ (BLYP). The Grimme's empirical formula ${ }^{50}$ was included to account for van der Waals interactions, generally missed by any GGA. The efficiency of such a computational set-up has been extensively benchmarked over the years on a wealth of aqueous systems ${ }^{51-54}$ and can be regarded as sufficiently accurate.

The porphyra-334 molecules that were found as stable, namely configurations 2 and 8 , were solvated in a periodically repeated supercell of liquid water at standard density $\left(1.0 \mathrm{gcm}^{-3}\right)$ and temperature $(300 \mathrm{~K})$. Given the encumbrance of these porphyra-334 structures, 120 and 125 water molecules were necessary to fill a cubic simulation cell of lateral size of $17.5 \AA$. The construction of these systems started by a classical molecular dynamics equilibration of the solvent water alone. Then, to insert the porphyra-334 molecular structures, a sufficient space was cleaved by eliminating all $\mathrm{H}_{2} \mathrm{O}$ molecules falling inside the van der Waals radii of the optimized porphyra334 geometries in their excited states (see figure S1 in the SI). Then the system was re-equilibrated for $10 \mathrm{ps}$ within a CPMD approach at $300 \mathrm{~K}$ in the canonical NVT ensemble with a Nosé-Hoover temperature control scheme. ${ }^{55-57}$ Initially, the structure of the porphyra-334 molecule was kept fixed to its excited state configuration, whereas the surrounding water was equilibrating. After the equilibration of the solvent, all constraints on the porphyra-334 geometry were released and the system was allowed to evolve dynamically on the underlying potential energy surface (PES). At this point, in order to eliminate any thermodynamical bias, the thermostats were suppressed and all simulations continued in the microcanonical NVE ensemble. Such a set-up accounts for the initial excitation of the solute upon sunlight adsorption, a process which is generally rapid enough to have little or no immediate influence on the surrounding solvent. Then, to reproduce the deactivation processes, initial velocities are attributed to the atoms of the porphyra-334 molecules to follow the non-radiative transition process that NVE simulations mimic, schematically illustrated in Figure 2.

The excited porphyra-334 molecule is expected to relax to the ground electronic state in a time 


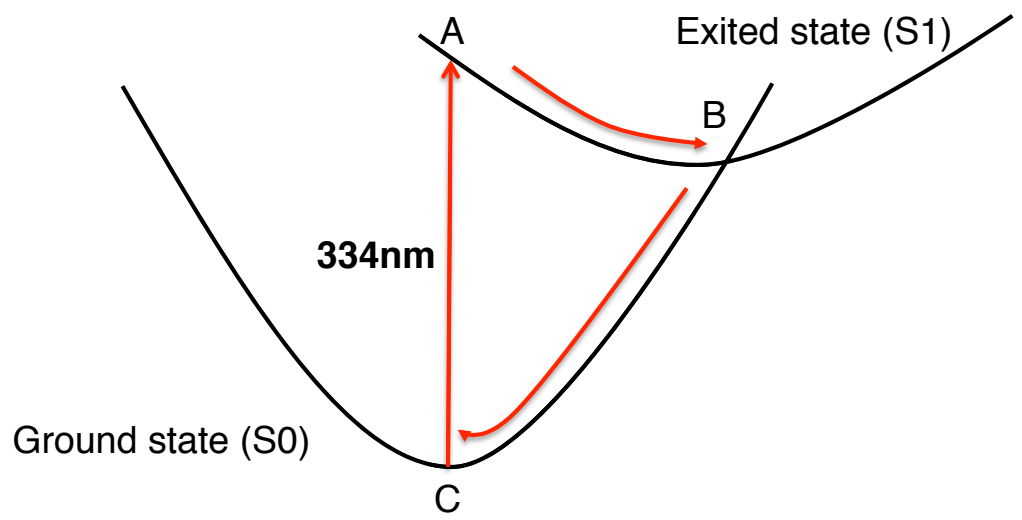

Figure 2: Schematic picture of the ground (S0) and excited (S1) states of the porphyra-334 molecule. The transition from $\mathrm{C}$ to $\mathrm{A}$ indicates the initial vertical transition from the ground to the excited state. The transition from A to B indicates the structural relaxation at the excited state. At the point $\mathrm{B}$, the system reverts to the ground state via a nonradiative transition. The transition from $\mathrm{B}$ to $\mathrm{C}$ indicates the structural relaxation at the ground state. At the point $\mathrm{C}$, the porphyra-334 acquires an excess of kinetic energy as a response to the adsorption of photons of wavelength of $334 \mathrm{~nm}$. Our dynamical simulations describe the process occurring for the transition from $\mathrm{B}$ to $\mathrm{C}$ and the subsequent relaxation in the range around point $\mathrm{C}$ on the ground state.

much shorter than the accompanying atomic relaxation, ${ }^{58,59}$ thus all the electron excitation energy is converted into an initial excess kinetic energies accumulated in the porphyra-334 molecule. Since CPMD simulations are done on the ground state electronic configuration, to reproduce such an initial excess kinetic energy, random velocities, sampled from a Maxwell-Boltzmann distribution corresponding to a typical $334 \mathrm{~nm}$ photon wavelength excitation, are attributed to the atoms of the porphyra-334 molecule to mimic these conditions. This amounts to an initial excess (kinetic) energy of $K=3.7 \mathrm{eV}$ redistributed among the porphyra-334 atomic constituents and, hence, resulting in a strong heating of about $624 \mathrm{~K}$ according to the standard formula $K=3 / 2 N k_{B} T$. Here, $k_{B}$ is clearly the Boltzmann constant and $T, N$ and $K$ indicate the temperature, the total number of atoms, and the total kinetic energy, respectively. Our CPMD simulations, then, account for the relaxation processes involved in the release of this extra kinetic energies of the porphyra-33 to the surrounding solvent water.

After an initial 10 ps equilibration of the solvent, with the porphyra-334 kept fixed, we continued the CPMD dynamical simulation observing a continuous breaking and formation of H-bonds 
around the solute molecule. This implies that the H-bond network around the porphyra-334 is highly dynamical and can rearrange on a ps time scale. In order to account for these different H-bond configurations and to increase the statistics, for both configurations 2 and 8 we selected at intervals of $10 \mathrm{ps}$ seven different configurations for each one. These time intervals are long enough to ensure that the sampled configurations are uncorrelated and the net result was a set of fourteen different configurations (seven for configuration 2 and seven for configuration 8) from which independent simulations of the porphyra-334 relaxation were started. To this aim, for each simulation a randomization of the initial velocities was done according to the procedure described above. Each one of these fourteen independent simulations lasted for $9 \mathrm{ps}$, a time that, although short, turned out to be sufficient to observe the total release of the excess kinetic energy initially stored in the porphyra-334. The process was then monitored and analyzed in term of mass-weighted autocorrelation functions for which details are given in the following subsection.

\section{Analysis of the Simulations Data}

To analyze the relaxation processes in which an energy transfer occurs from the porphyra-334 molecule to the surrounding solvating water, we resorted to the methodology proposed by Yagasaki and Saito. ${ }^{60-62}$ Such a procedure allows for a detailed inspection of the evolution in time of the kinetic energy transfers from the heated porphyra-334 to the solvent. Specifically, the extra kinetic energies accounting for the deexcitation of the porphyra-334 is partitioned into separate contributions, namely the intermolecular motion, the internal rotation, and the vibrational modes. Explicitly, the excess kinetic energy is written as

$$
\Delta C\left(t^{\prime}, T\right)=\frac{1}{2} \sum_{i} m_{i}\left\langle\boldsymbol{v}_{i}\left(t^{\prime}+T\right) \boldsymbol{v}_{i}(T)\right\rangle^{N E}-\frac{1}{2} \sum_{i} m_{i}\left\langle\boldsymbol{v}_{i}\left(t^{\prime}+T\right) \boldsymbol{v}_{i}(T)\right\rangle^{E Q}
$$

The superscript "NE" (non-equilibrium) indicates the ongoing relaxation process in which extra kinetic energy of the porphyra-334 molecule is gradually transferred to surrounding $\mathrm{H}_{2} \mathrm{O}$ molecules. The superscript "EQ", instead, refers to the dynamics in which both the porphyra-334 
and the solvating water are at the equilibrium, i. e. the system is an aqueous solution at $300 \mathrm{~K}$ in the present case. The mass and the velocity vector of the $i$ th atom are indicated as $m_{i}$ and $\boldsymbol{v}_{i}$, respectively. $T$ is the selected waiting time starting from which the calculation is done and $t^{\prime}$ is the running variable indicating the time evolution from $T$ onward. Hence, the first and second terms in the left-hand part of equation (1) are not directly kinetic energies (although resembling) but mass-weighted auto-correlation functions. A real Fourier transform, i. e. making use of the cosine only, gives

$$
\begin{aligned}
\Delta C\left(\omega_{t^{\prime}}, T\right) & =\frac{1}{\pi} \int_{0}^{\infty} d t^{\prime} \cos \left(\omega_{t^{\prime}} t^{\prime}\right) \sum_{i} m_{i}\left\langle\boldsymbol{v}_{i}\left(t^{\prime}+T\right) \boldsymbol{v}_{i}(T)\right\rangle^{N E} \\
& -\frac{1}{\pi} \int_{0}^{\infty} d t^{\prime} \cos \left(\omega_{t^{\prime}} t^{\prime}\right) \sum_{i} m_{i}\left\langle\boldsymbol{v}_{i}\left(t^{\prime}+T\right) \boldsymbol{v}_{i}(T)\right\rangle^{E Q}
\end{aligned}
$$

This equation can be interpreted as the frequency dependent difference between the kinetic energies of the non-equilibrated dynamics of the solvated excited porphyra-334 and the one of the equilibrated system. The above difference of frequency dependent kinetic energies can then be splitted into two separate contributions,

$$
\Delta C\left(\omega_{t^{\prime}}, T\right)=\Delta C^{t r a}\left(\omega_{t^{\prime}}, T\right)+\Delta C^{r o t}\left(\omega_{t^{\prime}}, T\right)
$$

where $\Delta C^{t r a}$ is the part accounting for the translational motion of the solvating $\mathrm{H}_{2} \mathrm{O}$ molecules, thus can be directly computed from the kinetic energies of the center of mass of the water molecules only. $\Delta C^{r o t}$ indicates, instead, the excess kinetic energy due to rotational and vibrational motions and can be calculated by subtracting the contributions. of the kinetic energies of the water molecules from the total kinetic energy of the whole solvent. On these grounds, we can define the partial excess kinetic energies (PEKEs) distributed by the five inter or intra molecular motion modes as 


$$
\begin{aligned}
& \Delta K^{H R}(T)=\int_{\omega_{\text {rot }}}^{\omega_{\text {vib }}} d \omega_{t^{\prime}} \Delta C^{r o t}\left(\omega_{t^{\prime}}, T\right), \\
& \Delta K^{L R}(T)=\int_{0}^{\omega_{r o t}} d \omega_{t^{\prime}} \Delta C^{r o t}\left(\omega_{t^{\prime}}, T\right), \\
& \Delta K^{V I B}(T)=\int_{\omega_{v i b}}^{\infty} d \omega_{t^{\prime}} \Delta C^{r o t}\left(\omega_{t^{\prime}}, T\right), \\
& \Delta K^{H T}(T)=\int_{\omega_{t r a}}^{\infty} d \omega_{t^{\prime}} \Delta C^{t r a}\left(\omega_{t^{\prime}}, T\right), \\
& \Delta K^{L T}(T)=\int_{0}^{\omega_{t r a}} d \omega_{t^{\prime}} \Delta C^{t r a}\left(\omega_{t^{\prime}}, T\right) .
\end{aligned}
$$

Here, $\omega_{\text {rot }}$ and $\omega_{\text {tra }}$ are set to 600 and $110 \mathrm{~cm}^{-1}$, respectively, according to the prescription proposed by Yagasaki and Saito. ${ }^{60-62}$ Analogously, $\omega_{v i b}$ is set to $1250 \mathrm{~cm}^{-1}$, a value able to fully separate the intramolecular vibrational contributions from the rotational ones. The type of motion represented by HR, LR, HT, and LT are sketched for clarity in Figure 3.

$$
\text { HR (y-axis rotation) LR (x-axis and z-axis rotations) }
$$

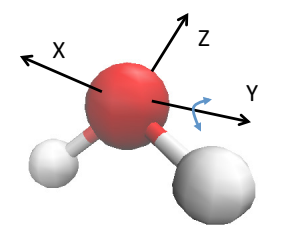

HT (hydrogen-bond stretch)

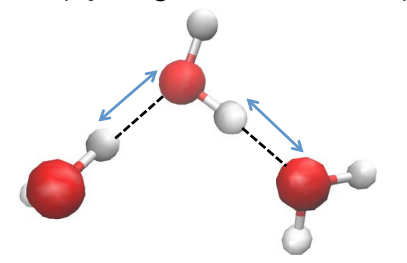

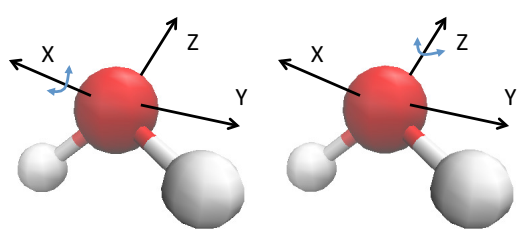

LT (hydrogen-bond bend)

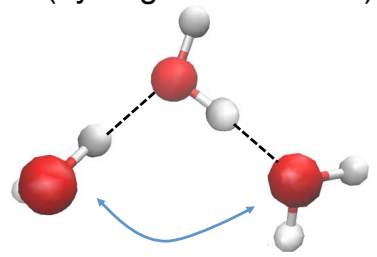

Figure 3: Schematic pictures of the four intermolecular motions.

With this algorithm, the excess kinetic energy is separated into five contributions (the fifth being the vibrational motion contribution) and these can discriminate between inter and intra molecular motions. From a strictly physical point of view, we have to remark that these PEKEs do not have the dimensions of a kinetic energy, but rather those of a kinetic energy ( $\mathrm{kcal} / \mathrm{mol})$ multiplied by a wave number $\left(\mathrm{cm}^{-1}\right)$. 


\section{Results and Discussions}

\section{Structural optimizations of the porphyra-334 in its ground and excited states}

To prepare the system for subsequent dynamical simulations, geometry optimizations of the porphyra334 molecule in its ground state were done within the DFT framework, by using a B3LYP functional to treat the exchange and correlation interactions. By assuming that the porphyra-334 is in a neutral charge state, the effects of a surrounding solvent were accounted for by a PCM model ${ }^{42,43}$ in all structural relaxations. As mentioned in the former paragraph, to account for all possible protonation states of hydroxy groups and nitrogen atoms of the porphyra-334, nine different structures were considered (see Figure 1). Our search for stable configurations has shown that the configurations labeled as 2 and 8 in Figure 1 are characterized by an energy difference of $0.15 \mathrm{eV}$, the lower reference value being the configuration 8 , and they are the most stable among all the possibilities analyzed. In all the cases in which an $\mathrm{NH}_{2}$ group is present in the molecule and when carboxyl ions are located in proximity of these $\mathrm{NH}_{2}$ groups, one of the two $\mathrm{H}$ of the $\mathrm{NH}_{2}$ moiety departs and migrates toward the carboxyl group nearby. The net result is that the geometries of configurations 3 and 4 in Figure 1 transform spontaneously into configurations 2 and 8, confirming again their better overall stability. Energetically speaking, above the two configurations 2 and 8 , we could locate the structure indicated as configuration 1. This specific structure is higher in energy by about $0.45 \mathrm{eV}$ with respect to configuration 8 and $0.30 \mathrm{eV}$ with respect to configuration 2 , the most stable being the former. Hence, on these grounds, we selected configurations 2 and 8 as possible porphyra-334 structures for subsequent excited state calculations and CPMD simulations. The optimized geometries of these two configurations are sketched in Figure 4.

Starting from the ground state optimized geometries for configurations 2 and 8, we re-optimized these two structures in their respective excited states at the CIS level by including a polarizable continuum model ${ }^{42,43}$ to account for solvent effects. In both cases singlet states were considered by imposing a total multiplicity $2 s+1=1$. The results in terms of structural changes between the excited and the ground state geometries are shown in Figure 5 


\section{(a) Configuration 2}

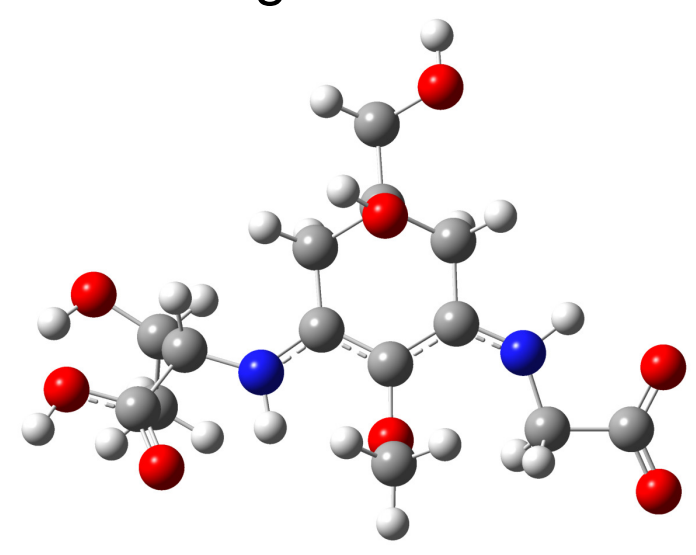

(b) Configuration 8

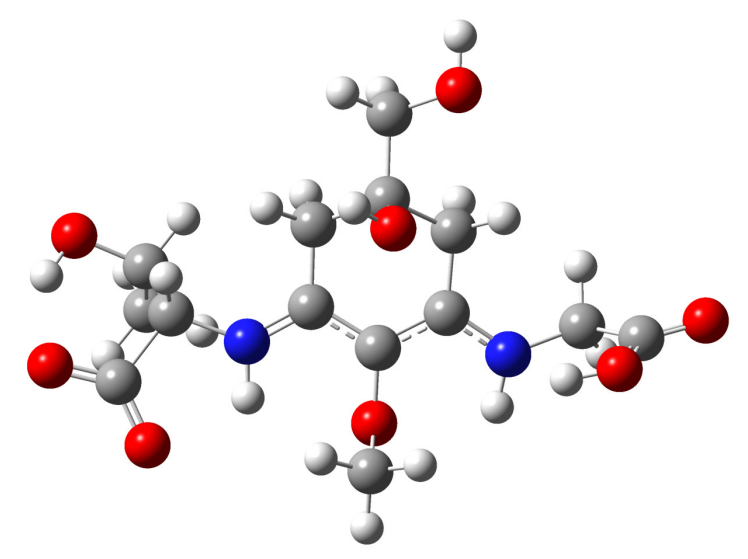

Figure 4: Optimized geometries of configurations 2 and 8. The labeling refers to Figure 1. The total energies of these two structures are separated by only $0.15 \mathrm{eV}$ and they represent the most stable minima. Oxygen, nitrogen, carbon, and hydrogen atoms are represented by red, blue, gray, and white spheres, respectively.

(a) Configuration 2

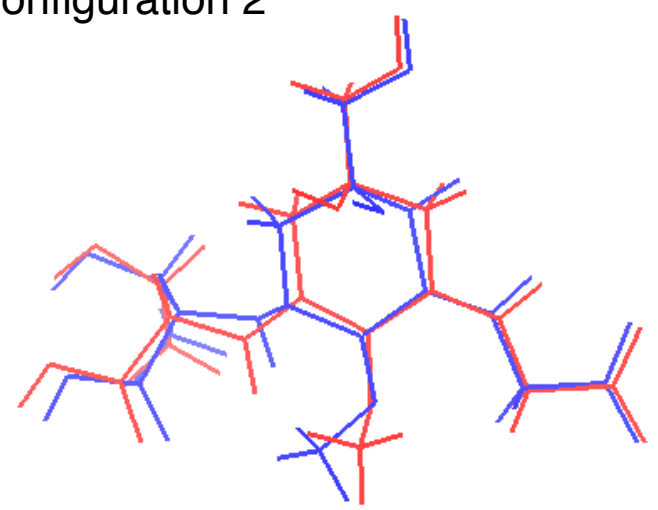

(b) Configuration 8

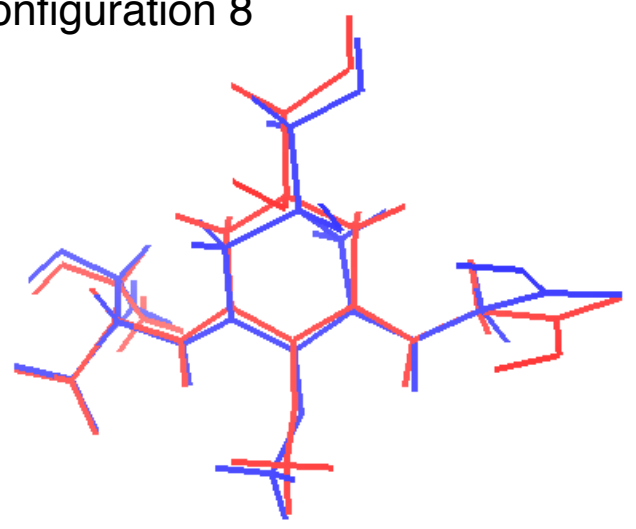

Figure 5: Superimposition of the ground (red) and excited (blue) state geometries of the porphyra334 molecule for the two most stable configurations. The root mean square deviations between the ground and the excited states are $0.51 \AA$ and $0.52 \AA$ for the configurations 2 and 8, respectively. 
The geometries of the excited states can be regarded as the ones corresponding to the point indicated as B in Figure 2 and they are starting structures for all subsequent simulations of the relaxation processes. A comparison of the excited and ground state geometries shows that the cyclohexane moieties become slightly bended upon electron excitation. Analogously, all carbon backbones around hydroxy and methyl groups show noticeable distortions with respect to the ground state configurations. The root mean square deviations (RSMDs) between the ground and the excited states turn out to be $0.51 \AA$ and $0.52 \AA$ for the configurations 2 and 8, respectively.

\section{First principles molecular dynamics simulations}

The excited state geometries of configurations 2 and 8 as provided by the CIS calculations were used as starting conformations for CPMD simulations. As explained in the section dedicated to the computational details, after solvating these structures in liquid water, we proceeded to a reequilibration of the solvent keeping the porphyra-334 fixed to the excited geometry. Once this equilibrium was attained, we attributed random velocities to the atoms of the porphyra-334 molecule sampled from a Maxwell-Boltzmann distribution corresponding to a temperature of $624 \mathrm{~K}$. This specific temperature, as explained above, accounts for the energy gained by the solute upon photon adsorption. With the two systems prepared in such a way, all constraints were released and dynamical simulations performed in the microcanonical NVE ensembles. According to the analysis described by equation (2), by setting the waiting time $T$ to zero when the equilibrium is reached we calculated the frequency dependent kinetic energy of the solvating water molecules, specifically the real Fourier transform of the mass-weighted velocity autocorrelation function. The result of this analysis is summarized in Figure 6. The disentangling of the frequency dependent kinetic energy from the contributions due to molecular translations, rotations and vibrations was done according to the definitions given in the computational methods section. This analysis shows that the frequency dependent kinetic energy of the translational motion has a pronounced peak centered at $100 \mathrm{~cm}^{-1}$ accompanied by a shoulder around $200 \mathrm{~cm}^{-1}$ (green line in Figure 6). The rotational contributions, on the other hand, are characterized by a peak centered at $600 \mathrm{~cm}^{-1}$. Peaks at 1600 
$\mathrm{cm}^{-1}$ and $3200 \mathrm{~cm}^{-1}$ represent, as usual, the bending and stretching molecular vibrations of liquid water and are clearly well separated by the selected threshold $\omega_{v i b}=1250 \mathrm{~cm}^{-1}$ discussed in the previous paragraph. These spectral distributions reproduce with appreciable accuracy the vibrational density of states provided by both classical simulations and experiments ${ }^{60}$ and represent a benchmark for our DFT-based CPMD simulations.

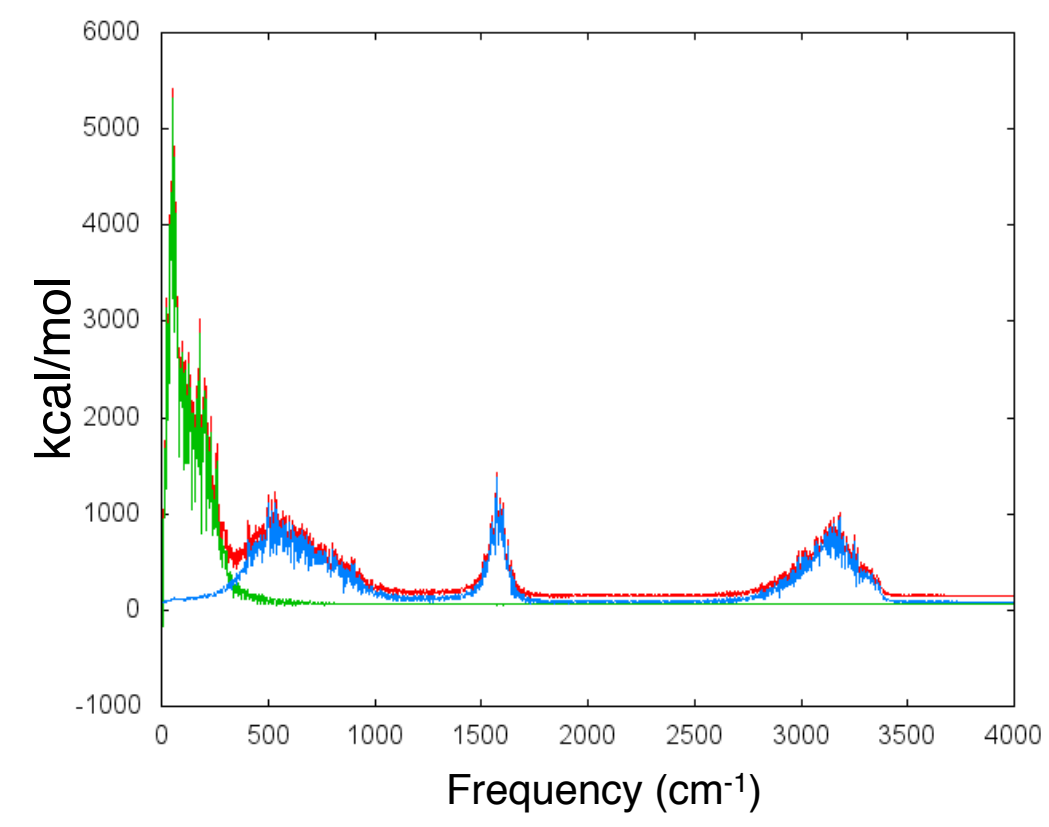

Figure 6: Results of the Fourier transform of the velocity autocorrelation functions for the equilibrated part of the simulations. The red line shows the spectrum of the total kinetic energy of the solvent water molecules. The green line show the contribution due to the translational motion. The blue line, instead, is the contributions due to both rotational and vibrational motions with the two peaks at $1600 \mathrm{~cm}^{-1}$ and $3200 \mathrm{~cm}^{-1}$ being the $\mathrm{H}_{2} \mathrm{O}$ molecular bending and stretching, respectively.

By changing the parameter $T$ in equation (2), we could follow the time evolution of the frequency dependent kinetic energy contribution. Since autocorrelation functions must be computed on each one of the $9 \mathrm{ps}$ independent simulations, we could extract frequency dependent kinetic energy contributions on a time scale of about $4 \mathrm{ps}$. When the system is at the equilibrium, it contributes clearly only to the second term of equation (2). The first term, instead, evolves in time as shown in panel (a) of Figure 7. Here, the most noticeable change is an increase of the low frequency part as a function of time because the collective molecular motion become increasingly important as long as the simulation proceeds, whereas the fast bending and stretching molecu- 
lar modes remains basically unaltered. In panel (b) of the same figure, we report the difference between the first term and the second term. This gives a more precise idea about the relative importance of the two terms as the time proceeds. Integrating this differences of kinetic energies with respect to the frequency, according to equations (4a)-(4e), we obtain the time evolutions of the PEKEs on which we focus in the next section.

(a)

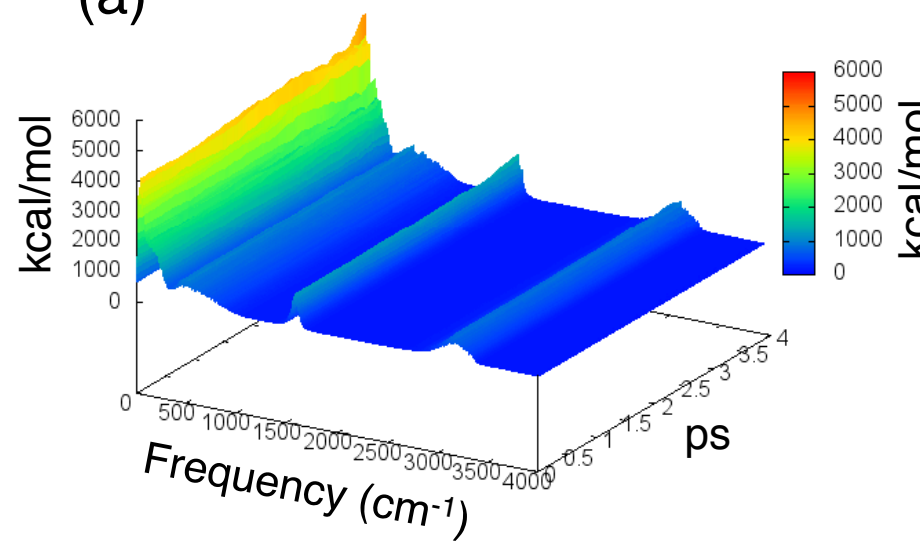

(b)

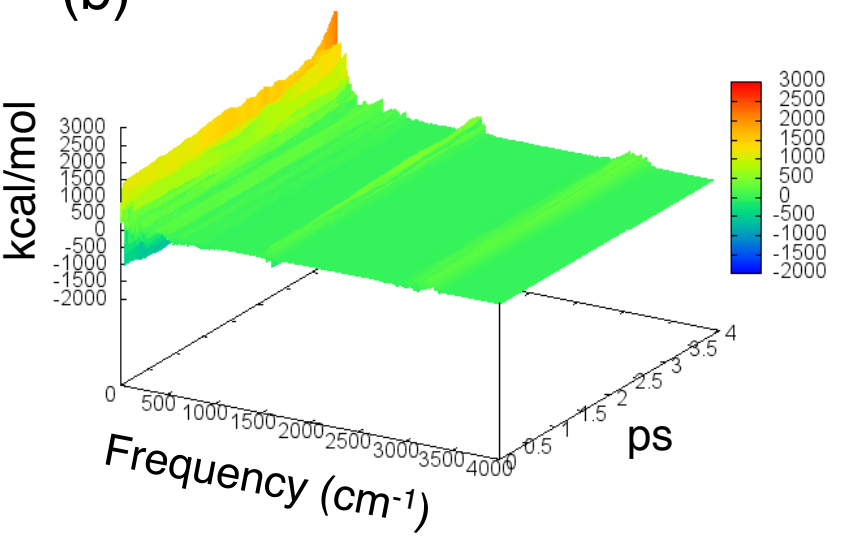

Figure 7: Evolution in time of the frequency dependent kinetic energy contribution (a) and analogous time evolution of the difference between non-equilibrated and equilibrated frequency dependent kinetic energy contributions (b) as expressed in equation 2. These results refer to the analysis of the dynamical evolution of the configuration 2 . The results of the configuration 8 are basically identical.

\section{Kinetic energy transfer from porphyra-334 to water and H-bond network evolution}

The time evolutions of the different PEKEs contributions during the dynamical evolution of configurations 2 and 8 are shown in Figures 8 and 10, respectively.

The result of the analysis of the various PEKEs contributions for the different initial H-bond configurations considered are shown in Figures S1 and S2 in the Supporting Information for both configurations 2 and 8 . More instructive is the statistical average extracted from these independent simulations. Specifically, the average trend indicates that the PEKE contribution due to the HT motion is the one that increases more and monotonically in both cases, thus making this H-bond 
(a)

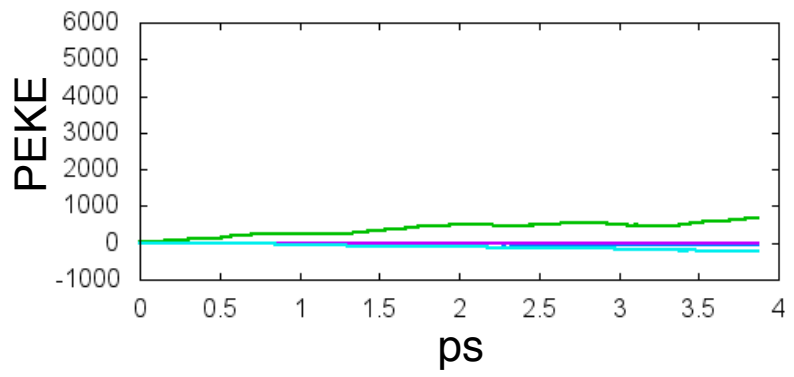

(b)

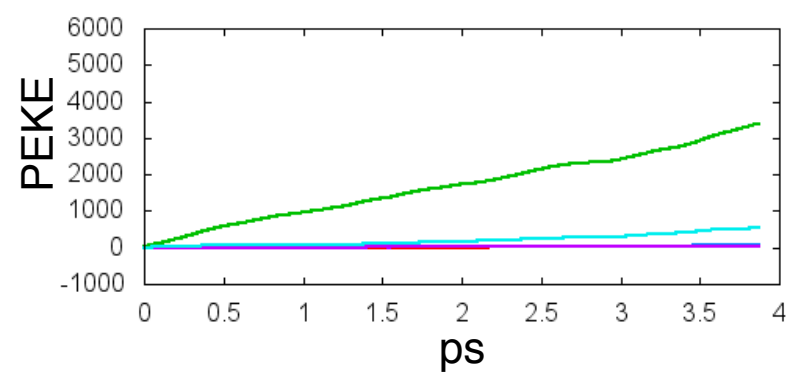

Figure 8: Time evolution of the various PEKEs contributions averaged over all the seven independent simulations. The red, green, blue, and pink lines indicate the contributions of the four LT, HT, LR, HR PEKEs functions, respectively. The cyan lines refer, instead, to the vibrational motions (see Figure 3). (a) and (b) are results of simulations using configuration 2 and 8, respectively.

stretching the preferred channel for the dissipation of the excess kinetic energy of the porphyra334 molecule. In fact, between the two configurations 2 and 8, the increase of the HT PEKE contributions as a function of time is remarkably larger in the latter case. Only minor or nearly negligible contributions can be appreciated by observing the trend of the PEKEs due to the LT, LR, HR, and vibrational contributions. It can be inferred that the rather large kinetic energy gained upon photoexcitation directly activates the higher translational modes, hence HT rather than the lower LT mode Most likely this is due to the fact that the HT mode can accept a larger amount of excess energy with respect to the other modes, namely LT, LR, and HR.

Since the HT motion involves the collective shrinking and elongation of intermolecular Hbonds, this dynamical change of the distance between $\mathrm{H}_{2} \mathrm{O}$ molecules can result in the breaking (and reforming) the local H-bond network which, in turn, stabilize the hydration shell of the porphyra-334 molecule. More precisely, a decrease in the number of H-bonds destabilizes temporarily the system and produces an increase in the potential energy of the solvent water. To make this statement more quantitative, we analyzed the evolution of the total number of H-bonds during all the 9 ps dynamical simulations, considering both the $\mathrm{H}$-bond network of the solvent and the $\mathrm{H}$ bonds of the solute with the surrounding water. To this aim, the $\mathrm{H}$-bonds between $\mathrm{H}_{2} \mathrm{O}$ molecules and between the solvent water and the porphyra-334 solute were monitored by using the Visual Molecular Dynamics (VMD) tool. ${ }^{63}$ A snapshot of the continuous H-bond network of the system 
is shown in the SI (Figure S1) and its local evolution along the dynamics is reported in panels (a) and (b) of Figure 9.

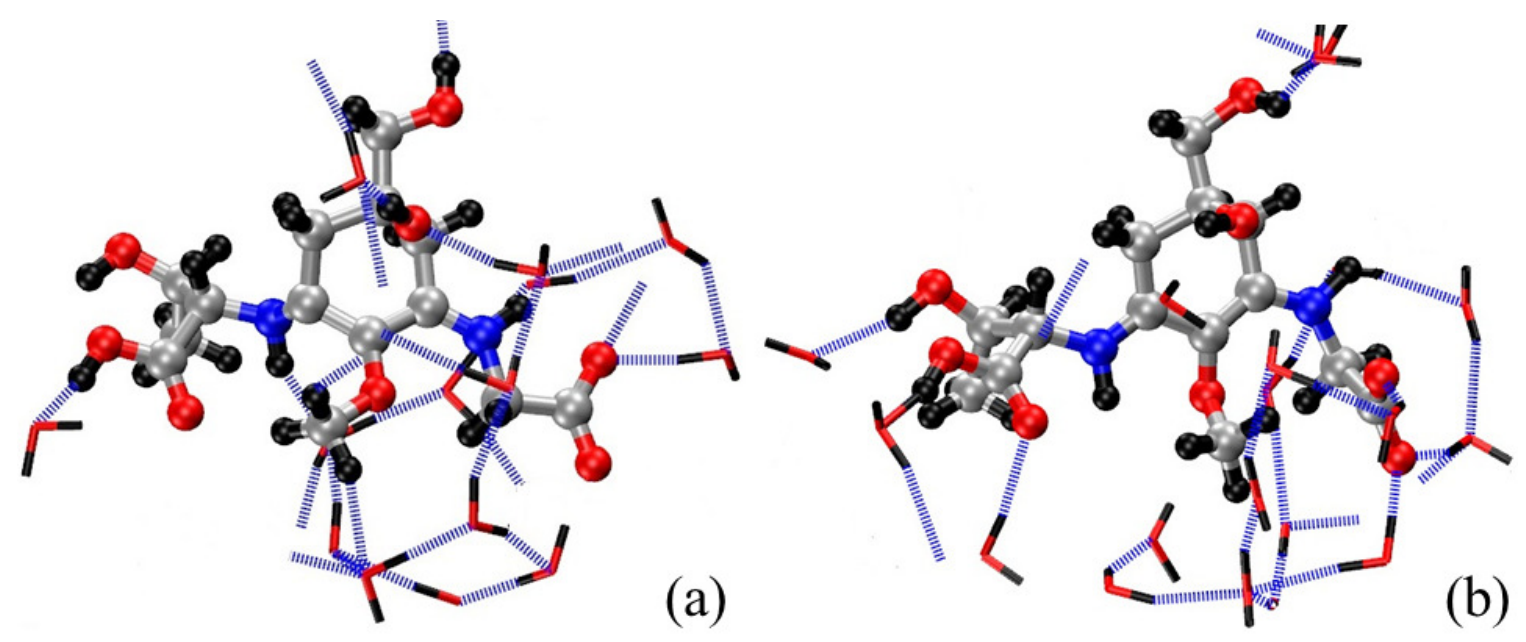

Figure 9: Evolution of the hydrogen bond network around the porphyra-334 molecule solvate din water for the configuration 2 structure. Panel (a) shows the hydrogen bonding of the solute with solvating water at the beginning of the simulation, whereas panel (b) shows the dynamical evolution at the end of the run where the equilibrium is attained. Red, blue, gray, and black balls and sticks indicate oxygen, nitrogen, carbon, and hydrogen, respectively, while hydrogen bonds are shown as dashed blue lines.

A simple visual inspection of Figure 9 shows that the number of $\mathrm{H}$-bond between solvent and solute is highly variable as the dynamics proceeds and this, in turn, influence the whole solvent. Such a process has been quantified by monitoring H-bond formation and disruption. For the solvent only, the result of this analysis is summarized in Figures (10)) for the systems whose simulations were started from configurations 2 and 8, respectively. This result represents the average over all the seven independent simulations. Details for each single simulations are reported in the SI, Figures S4 and S5.

By looking at the trend of the rolling average, in the case of simulations started from the prophyra-334 in the configuration 2 we can observe a gradual decreases in the number of H-bonds from an initial value of $\sim 75$ to a final value of $\sim 60$. An analogous trend characterized also the evolution for the system started from configuration 8, where, again, a drop in the number of $\mathrm{H}_{-}$ bonds can be observed as the simulation progresses. The overall conclusion that can be drawn is that the activation of the intermolecular HT motion destabilized and disrupts to some extent the 
(a)

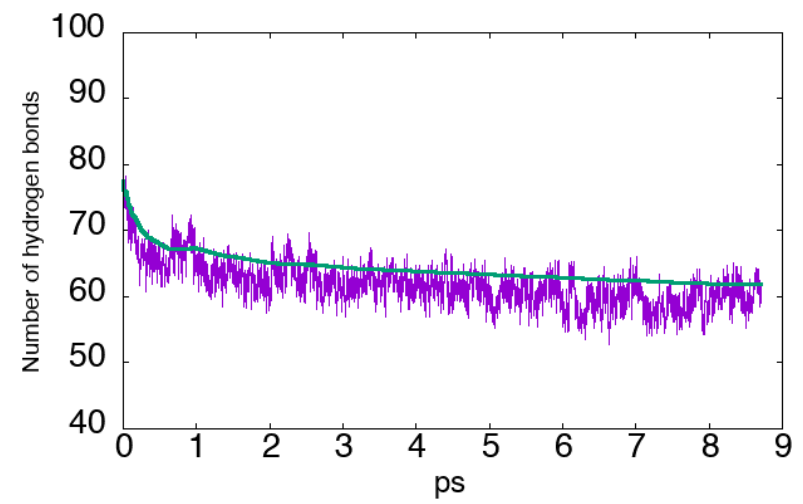

(b)

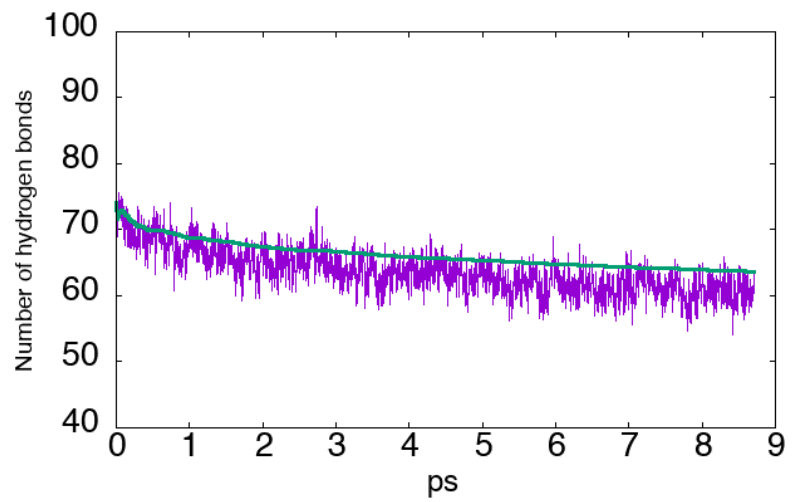

Figure 10: Time evolution of the number of hydrogen bonds between water molecules averaged over all the seven independent simulations. The purple lines indicate the evolution in time of the total numbers of hydrogen bonds while the green lines represent the rolling averages. (a) and (b) are results of simulations using configuration 2 and 8 , respectively.

(a)

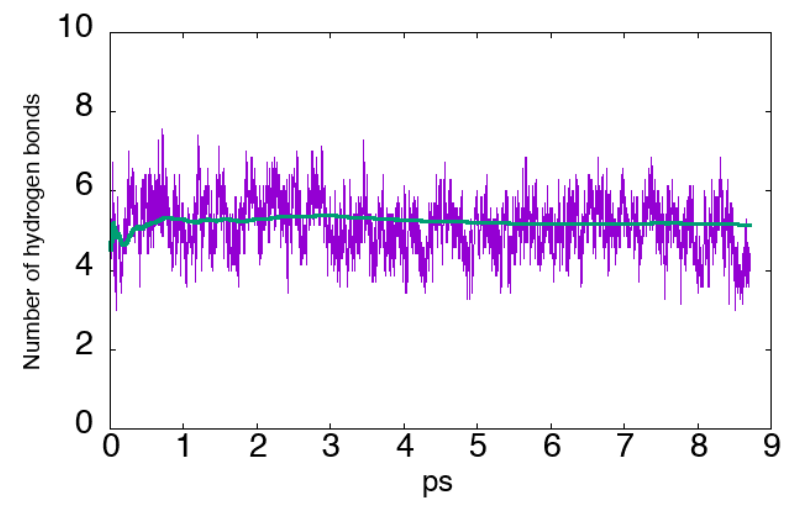

(b)

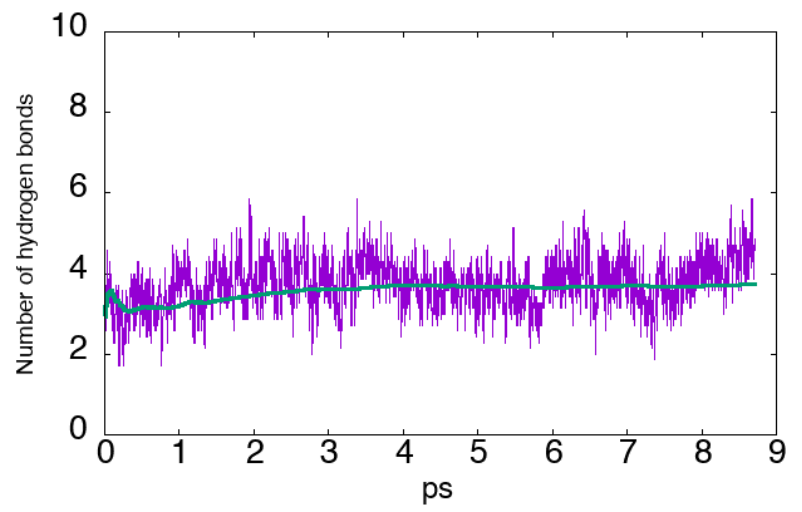

Figure 11: Time evolution of the numbers of the hydrogen bonds between porphyra-334 and surrounding water molecules averaged over all the seven independent simulations. The purple lines indicate the total instantaneous number of hydrogen bonds, whereas the green lines are the rolling average computed from these time evolutions. (a) and (b) are results of simulations using configuration 2 and 8 , respectively. 
H-bond network of the solvent around the porphyra-334 molecule. This effects is, as expected, more pronounced in the vicinity of the solute and a clear result of the release of the excess kinetic energy to the hydrating $\mathrm{H}_{2} \mathrm{O}$ molecules around. Yet, such a destabilization affects just the $\mathrm{H}$-bond topology and does not involve any water molecule dissociation at any stage or distance from the excited solute. The related electronic structure changes on the porphyra-334 molecule induced by the H-bond environment evolution was quantified in terms of Mulliken population analysis. This inspection has shown that all $\mathrm{H}$ atoms belonging to hydroxy groups or bound to $\mathrm{N}$ atoms carry a Mulliken charge of $0.30 \mathrm{e}$, whereas all other hydrogen atoms are characterized by charges of $0.25 e$. The picture that emerges from these values is that $-\mathrm{OH}$ and $-\mathrm{NH}$ moieties are the most polarized groups of the porphyra-334 and, as such, those are prone to form tight H-bonds with the solvating water. Indeed, a monitoring of these $\mathrm{H}$-bonds along all the trajectories of the independent simulations has shown that these never undergo breaking at least on the 9 ps time scale of each run. Moreover, the - $\mathrm{OH}$ hydroxy groups can form more than one $\mathrm{H}$-bond with the $\mathrm{H}$ atom pointing toward an $\mathrm{O}$ atom of a water molecule in the vicinity and the $\mathrm{O}$ atom pointing with its lone pairs at $\mathrm{H}$ atoms of other $\mathrm{H}_{2} \mathrm{O}$ molecules sufficiently close. Another feature worthy of note is the fact that the $-\mathrm{COO}^{-}$carboxylate ion which is part of the porphyra-334 was able to make remarkably stable $\mathrm{H}$-bonds with nearby $\mathrm{H}_{2} \mathrm{O}$ molecules.

The complementary part of this analysis is represented by the inspection of the number of $\mathrm{H}$ bonds between the porphyra-334 and the solvent water along the 9 ps simulations. The evolution of this number allows to quantify the changes that the hydration shell of the porphyra-334 undergoes in the energy release process and the fact of having independent simulations allows for a refinement of the statistics.

The result of this analysis is summarized in Figure 11 for both configurations 2 and 8 . The rolling averages, shown as a green lines in both panels, indicate that the numbers of H-bonds are kept nearly constant during the energy release process and, on average, equal to 5 and 3 in the cases of configurations 2 and 8, respectively. Details concerning the separate contributions in the number of H-bond for each simulation are shown in Figure S6 and S7 in the SI. The clear 
picture that emerges is that the H-bonding of the solute molecule with the surrounding solvent is pretty stable. As a consequence, this allows for a continuous transfer of the excess kinetic energy from the (excited) porphyra-334 to the surrounding environmental water. Although the red lines, indicating the instantaneous counting, look rather scattered and can occasionally decrease until 1 , the hydration shell of the solute is just instantaneously disrupted but immediately restored, thus keeping the connection solute-solvent always active and never hindering permanently the excess energy transfer process. Analogously, our results shows that a single porphyra-334 molecule can increase temporarily the number of H-bonds with the solvent water up to a maximum of ten, thus opening additional channels to the kinetic energy transfer.

By summarizing the message of the two analyses, we can infer that only the H-bond network of the solvent is disrupted, since only in the case of the solvent a clear decreasing trend in the number of H-bonds has been found. The destabilization of the water H-bond network propagates away from the porphyra-334 molecule and is solely the result of the excess kinetic energy release. At the same time, the tightness of the $\mathrm{H}$-bonds of the porphyra-334 with the $\mathrm{H}_{2} \mathrm{O}$ molecules of its own first hydration shell represent a key feature to promote an energy transfer sufficiently smooth to prevent chemical bond breaking and molecular dissociation of both the solute and the solvent. hence, we can conclude that the activated HT motion of the water surrounding the excited porphyra-334 affects directly the disruption of the hydrogen bond network. This effect can be regarded as the

fundamental explanation about the mechanism along which the excess of energy accumulated by the photosensitive component of seaweeds upon electron excitation is released into the surrounding water and how the inherent hydrogen bond network of liquid water responds to this large energy transfer.

\section{Conclusions}

After determining the possible stable configurations of a porphyra-334 molecule in its ground state, we inspected the structural changes occurring upon photon adsorption via precise quantum chemi- 
cal calculations at the CIS level. On these grounds, first principles molecular dynamics simulations were used to inspect in detail the release of the excess kinetic energy accumulated in the excited porphyra-334 toward the surrounding environment represented by water. Our simulations were able to reproduce the nonradiative transition processes involved in such an energy release, clarifying both the mechanism and the preferred channel along which such an excess energy is released when the porphyra-334 relaxes back to its ground state. The disentangling of the different contribution in terms of partial excess kinetic energies attributed to the various collective modes of the solvent has shown that the main pathway along which the kinetic energy transfer occurs is the hydrogen bonds stretching (HT mode) among water molecules. Indeed, this is the mode that induces a minimal perturbation and distortion of the continuous H-bond network, as opposed to molecular rotations (HR, LR) and bending (LT). These HT stretching mode can result in instantaneous breaking, but an equally instantaneous reformation of existing H-bonds within the continuous net-

work of the tetrahedrally coordinated $\mathrm{H}_{2} \mathrm{O}$ constituents of the solvent. A precise monitoring of the number of H-bonds during the dynamical evolution of the system toward the equilibrium has shown that this number decreases gradually by $\sim 30$ in the solvating water, whereas the number of H-bonds between the porphyra-334 and the surrounding water is basically constant all along the energy release process. Occasionally, these H-bonds can fluctuate and their increase, although transitory, favor further the release process. These results provide a clear microscopic picture of the processes responsible for the de-excitation of porphyra-334 and represent a detailed answer to the question concerning the way in which seaweeds get rid of the excess energy without provoking neither their own destabilization not the dissociation of water molecules present in their natural environment.

\section{Acknowledgement}

We are deeply indebted to Prof. Shinji Saito at Institute for Molecular Science (Japan) for fruitful discussions and advices. This research was supported by JSPS KAKENHI (No. 25288012), Elements Strategy Initiative to Form Core Research Center (since 2012), and by MEXT, Japan. 
We thank computer facilities of ISSP and ITC at The University of Tokyo, RCCS at Okazaki, Japan, and the K computer (Project ID: hp160046) at AICS. A part of the calculations was performed on the HOKUSAI GreatWave system in RIKEN. M.B. is grateful to Pôle HPC and Equipex Equip@Meso at the University of Strasbourg, Grand Equipement National de Calcul Intensif (GENCI) under allocation DARI-A2 A0020906092.

\section{Supporting Information Available}

A listing of the contents of each file supplied as Supporting Information should be included. For instructions on what should be included in the Supporting Information as well as how to prepare this material for publications, refer to the journal's Instructions for Authors.

The following files are available free of charge.

- Filename: brief description

- Filename: brief description

This material is available free of charge via the Internet at http://pubs . acs . org/.

\section{References}

(1) Torres, A.; Enk, C. D.; Hochberg, M.; Srebnik, M. Photochem. Photobiol. Sci. 2006, 5, 432-435.

(2) Gupta, V.; Ratha, S. K.; Sood, A.; Chaudhary, V.; Prasanna, R. Algal Research 2013, 2, 79-97.

(3) Chuang, L. -F.; Chou, H. -N.; Sung, P. -J. Mar. Drugs 2014, 12, 4732-4740.

(4) Garcia-Pichel, F.; Wingard, C. E.; Castenholz, R. W. Appl. Environ. Microbiol. 1993, 59, 170-176. 
(5) Rezanka, T; Temina, M; Tolstikov, A. G.; Dembitsky, V. M. Folia Microbiologica 2004, 49, $339-352$.

(6) Tsujino, I. Bulletin of Faculty of Fisheries Hokkaido University 1961, 12, 59-65.

(7) Favre-Bonvin, J.; Arpin, N; Brevard, C. Can. J. Chemistry 1976, 54, $1105-$.

(8) Carreto, J. I.; Carignan, M. O. Mar. Drugs 2011, 9, 387-.

(9) Oren, A.; Gunde-Cimerman, N. FEMS Microbiol. Lett. 2007, 269, 1-.

(10) Wada, N.; Sakamoto, T.; Matsugo, S. Antioxidants 2015, 4, 603-.

(11) Vale, P. Photochemistry and Photobiology 2016, 92, 264-.

(12) Laurion, I.; Lami, A,; Sommaruga, R. Aquatic. Microb. Ecology 2002, 26, 283-294.

(13) Bathia, A.; Garg, A.; Sharna, K.; Kumar, S.; Sharma, A.; Purohit, A. P. Pharmacogn Rev. 2011, 5, 138-146.

(14) Misonou, T.; Nishio, H.; Tokitomo, Y.; Inoue, Y.; Hori, H.; Sakurai T. Proceedings of 3rd International Scientific-practical conference on Marine coastal ecosystem, 2009 pp.351-352. (Vladivostok)

(15) Whitehead, K; Hedges, J. I. J. Photochem. Photobiol. B 2005, 80, 115-.

(16) Conde, F. R.; Churio, M. S.; Previtali, C. M. J. Photochem. Photobiol. B 2000, 56, 139-144.

(17) Conde, F. R.; Churio, M. S.; Previtali, C. M. Photoch. Photobio. Sci. 2004, 3, 960-.

(18) Conde, F. R.; Churio, M. S.; Previtali, C. M. Photoch. Photobio. Sci. 2007, 6, 669-.

(19) Inoue, Y.; Hori, H.; Sakurai, T.; Tokitomo, Y.; Saito, J.; Misonou, T. Opt. Rev. 2002, 9, 75-.

(20) Marx, D; Hutter, J. Ab initio Molecular Dynamics: Basic Theory and Advanced Methods, Cambridge University Press 2009. 
(21) Marx, D. ChemPhysChem 2006, 7, 1848-1870.

(22) Sprik, M. J. Phys. Chem. 1991, 95, 2283-2291.

(23) Sprik, M. J. Phys. :Condens. Matt. 2000, 12, A161-A163.

(24) Silvestrelli, P. L.; Parrinello, M. J. Chem. Phys. 1999, 111, 3572-3580.

(25) Silvestrelli, P. L. J. Phys. Chem. B 2009, 113, 10728-10731.

(26) Koizumi, K.; Boero, M.; Shigeta, Y.; Oshiyama, A. Phys. Rev. B 2012, 85, 205314.

(27) Koizumi, K.; Boero, M.; Shigeta, Y.; Oshiyama, A. J. Phys. Chem. Lett. 2013, 4, 1592-1596.

(28) Koizumi, K.; Nobusada, K.; Boero, M. Chem. Eur. J. 2015, 22, 5181-5188.

(29) Koizumi, K.; Nobusada, K.; Boero, M. J. Phys. Chem. C 2015, 119, 15421-15427.

(30) Koizumi, K.; Nobusada, K.; Boero, M. Phys. Chem. Chem. Phys. 2016, 18, 20708-20712.

(31) Koizumi, K.; Nobusada, K.; Boero, M. Chem. Eur. J. 2017, 23, 1531-1538.

(32) Boero, M.; Ikeda, T.; Ito, E.; Terakura, K. J. Am. Chem. Soc. 2006, 128, 16798-16807.

(33) Kamiya, K.; Boero, M.; Tateno, M.; Shiraishi, K.; Oshiyama, A. J. Am. Chem. Soc. 2007, 129, 9663-9673.

(34) Gervasio, F. L.; Boero, M.; Parrinello, M. Angew. Chem. Int. Ed. 2006, 45, 5606-5609.

(35) Kamiya, K.; Baba, T.; Boero, M.; Matsui, T.; Negoro, S.; Shigeta, Y. J. Phys. Chem. Lett. 2014, 5, 1210-1216.

(36) Hohenberg, P.; Kohn, W. Phys. Rev. 1964, 136, B864-B871.

(37) Kohn, W.; Sham, L. J. Phys. Rev. 1965, 140, A1133-A1138.

(38) Becke, A. D. J. Chem. Phys. 1993, 98, 5648-. 
(39) Becke, A. D. Phys. Rev. A 1988, 38, 3098-.

(40) Lee, C.; Yang, W.; Parr, R. G. Phys. Rev. B 1988, 37, 785-

(41) Hariharan, P. J.; Pople, J. A. Theor. Chem. Acta. 1973, 28, 213-.

(42) Scalmani, G.; Frisch, M. J. J. Chem. Phys. 2010, 132, 114110-.

(43) Miertus, S.; Scrocco, E.; Tomasi, J. Chem. Phys. 1981, 55, $117-$

(44) Frisch, M. J.; Trucks, G. W.; Schlegel, H. B.; Scuseria, G. E.; Robb, M. A.; Cheeseman, J. R.; Scalmani, G.; Barone, V.; Mennucci, B.; Petersson, G. A.; Nakatsuji, H.; Caricato, M.; Li, X.; Hratchian, H. P.; Izmaylov, A. F.; Bloino, J.; Zheng, G.; Sonnenberg, J. L.; Hada, M.; Ehara, M.; Toyota, K.; Fukuda, R.; Hasegawa, J.; Ishida, M.; Nakajima, T.; Honda, Y.; Kitao, O.; Nakai, H.; Vreven, T.; Montgomery, J. A., Jr.; Peralta, J. E.; Ogliaro, F.; Bearpark, M.; Heyd, J. J.; Brothers, E.; Kudin, K. N.; Staroverov, V. N.; Kobayashi, R.; Normand, J.; Raghavachari, K.; Rendell, A.; Burant, J. C.; Iyengar, S. S.; Tomasi, J.; Cossi, M.; Rega, N.; Millam, J. M.; Klene, M.; Knox, J. E.; Cross, J. B.; Bakken, V.; Adamo, C.; Jaramillo, J.; Gomperts, R.; Stratmann, R. E.; Yazyev, O.; Austin, A. J.; Cammi, R.; Pomelli, C.; Ochterski, J. W.; Martin, R. L.; Morokuma, K.; Zakrzewski, V. G.; Voth, G. A.; Salvador, P.; Dannenberg, J. J.; Dapprich, S.; Daniels, A. D.; Farkas, Ö .; Foresman, J. B.; Ortiz, J. V.; Cioslowski, J.; Fox, D. J. Gaussian 09, Revision E.01, Gaussian, Inc., Wallingford CT, 2009.

(45) Car, R.; Parrinello, M. Phys. Rev. Lett. 1985, 55, 2471-2474.

(46) CPMD, http://www.cpmd.org/, Copyright IBM Corp 1990-2017, Copyright MPI für Festkörperforschung Stuttgart 1997-2001.

(47) Goedecker, S.; Teter, M.; Hutter, J. Phys. Rev. B 1996, 54, 1703-1710.

(48) Hartwigen, C.; Goedecker, S.; Hutter, J. Phys. Rev. B 1998, 58, 3641-3662.

(49) Krack, M. Theor. Chem. Acc. 2005, 114, 145-152. 
(50) Grimme, S. J. Comput. Chem. 2004, 25, 1463-1473.

(51) Boero, M.; Terakura, K.; Ikeshoji, T.; Liew, C. C.; Parrinello, M. J. Chem. Phys. 2001, 115, 2219-2227.

(52) Sprik, M.; Hutter, J.; Parrinello, M. J. Chem. Phys. 1996, 105, 1142-1152.

(53) Silvestrelli, P. L.; Parrinello, M. Phys. Rev. Lett. 1999, 82, 3308-3311.

(54) Marx, D.; Tuckerman, M. E.; Hutter, J.; Parrinello, M. Nature 1999, 397, 601-604.

(55) Nosé, S. Mol. Phys. 1984, 52, 255-268.

(56) Nosé, S. J. Chem. Phys. 1984, 81, 511-519.

(57) Hoover, W. G. Phys. Rev. A 1985, 31, 1695-1697.

(58) Doltsinis, N. L.; Marx, D. J. Theor. Comput. Chem. 2002, 1, 319-349.

(59) Boero, M.; Oshiyama, A.; Silvestrelli, P. L.; Murakami, K. Appl. Phys. Lett. 2005, 86, 201910 .

(60) Yagasaki, T.; Saito, S. J. Chem. Phys., 2011, 134, 184503.

(61) Yagasaki, T.; Saito, S. J. Chem. Phys., 2011, 135, 244511.

(62) Imoto, S.; Xantheas, S. S.; Saito, S. J. Phys. Chem. B, 2015, 119, 11068.

(63) Humphrey, W.; Dalke, A.; Schulten, K., J. Molec. Graphics, 1996, 14, 33. 